\title{
Acrylamide in fried potato products
}

\begin{abstract}
High acrylamide levels have been detected in fried potato products. Dietary intake studies observed large differences in acrylamide between single foodstuffs, within food categories, and within batches of similarly processed products. FAO/WHO emphasized that causes of variation need to be understood to develop effective measures. Particularly at Food Service Establishments acrylamide formation combined with wide consumption of French fries comprises a risk. This chapter illustrates the large variation in acrylamide precursors and acrylamide in fried potato products, and analyzes potential causes of variation in French fries preparation, particularly, food handlers practices as variation source is demonstrated. The variable effect of mitigation measures in FSE has been discussed. Acrylamide risk assessment should consider people impact on variable acrylamide in potato-based products as risk factor.
\end{abstract}

Keywords: Acrylamide; Food handlers; Food service establishments; French fries; Mitigation measure 